\title{
COSMIC EVOLUTION WITH EARLY AND LATE ACCELERATION INSPIRED BY DUAL NATURE OF THE RICCI SCALAR CURVATURE
}

\author{
S.K.Srivastava \\ Department of Mathematics, North Eastern Hill University, \\ NEHU Campus,Shillong - 793022 ( INDIA ) \\ e-mail:srivastava@nehu.ac.in ; sushil@iucaa.ernet.in
}

\begin{abstract}
It is found that dark energy emerges spontaneously from the higherderivative gravity. According to cosmological scenario, obtained here, the universe inflates for $\sim 10^{-37}$ sec. in the beginning and late universe accelerates after 8.58Gyrs. During the long intermediate period, it decelerates driven by radiation and subsequently by matter. Emerged gravitational dark energy mimics quintessence and its density falls by 115 orders from its initial value $2.58 \times 10^{68} \mathrm{GeV}^{4}$ to current value $2.19 \times 10^{-47} \mathrm{GeV}^{4}$. Key Words : Higher-drivative gravity, dark energy, inflation and late cosmic acceleration.
\end{abstract}

PACS Nos. : $98.80 \mathrm{Hw}, 98.80 .-\mathrm{k}, 98.80 . \mathrm{Cq}$ 


\section{Introduction}

In the past few years, astronomical observations have revolutionarized the cosmolgy. Consequences of combined analysis of Ia Supernovae (SNe Ia) observations [1, 2], galaxy cluster measurements [3] and cosmic microwave background (CMB) data [4] show dominance of dark energy (DE) in the present universe causing cosmic acceleration. Acceleration is observed at a very small red-shift showing that it is a recent phenomenon in the late universe. The 16 type Ia Supernovae, observed by Hubble Space Telescope (HST)[5], further modifies earlier astronomical results and provides conclusive evidence supporting deceleration prior to cosmic acceleration caused by DE in the recent past. DE is realized with negative pressure and positive energy density violating strong energy condition (SEC) [6]. Violation of SEC gives reverse gravitational effect. It is due to this effect, universe gets a jerk and transition from deceleration to acceleration takes place. Of late, phantom appeared as a potential DE candidate in this arena, which violates weak energy condition (WEC) too $[7,8]$. Though DE dominates at present, but the present value of dark energy density (DED) is very low $\sim 2.18 \times 10^{-47} \mathrm{GeV}^{4}$. In the early universe, it is found very high. For example, at Planck scale DED is found to be $1.18 \times 10^{75} \mathrm{GeV}^{4}$, at GUT (grand unified theory) scale it is $\sim 10^{23} \mathrm{GeV}^{4}$ and at QCD scale it is $\sim 10^{-6} \mathrm{GeV}^{4}$. The simplest DE candidate is constant $\Lambda$, but it has a serious problem of extreme fine-tuning to satisfy the current value of DED. Alternatively, to explain decay of DED from a very high value to present extremely small value, many dynamical models were suggested, where $\Lambda$ varies slowly with cosmic time $(t)[9,10]$. Apart 
from dynamical $\Lambda(t)$, many hydrodynamic models with or without dissipative pressure were proposed, where barotropic fluid is the source of DE [10]. In the literature, Chaplygin gas (CG) as well as generalzed Chaplygin gas (GCG) have been considered as possible DE sources due to negative pressure of these $[8,11,12,13]$. In field-theoretic models, most natural ones are scalar field models. Apart from quintessence and phantom $[6,7,8]$, other scalar field models are k-essence [14] and tachyon [15]. Other than these approaches, some authors have considered modified gravitational action by adding a function $f(R)$ ( $R$ being the Ricci scalar curvature) to Einstein-Hilbert lagrangian, where $f(R)$ provides a gravitational alternative for dark energy causing late-time acceleration of the universe. In $[16,17], f(R)=1 / R$, but it has the problem of instabilities of gravitationally bound objects [18]. In [19], $f(R)$ has the form $a R^{m}+b R^{-n}$ (where $a, b, m, n$ are real numbers). $R$ with positive power is dominant in the early universe giving power-law inflation for $1<m<2$. $R$ with negative power dominates in the late universe when curvature is small and gives late-time acceleration.In other attempts adopting this approach $f(R)$ is taken as $\ln R$ in [20] and $\operatorname{Tr}(1 / R)$ in [21] Some other modifications are also suggested in $[22,23]$. Recently, an arbitrary fuction of Gauss-Bonnet term has been considered for $f(R)$ as gravitational dark energy [24]. Thus, in the past few years, many models for non-gravitational and gravitational DE have been proposed to explain accelerated universe.

All these models are phenomenological in the sense that, in these models, an idea of DE is introduced a priori. But it is interesting to have a model, where DED emerges spontaneously from a basic theory. With this motivation, here DED is obtained from the gravitational 
sector, where a term $\alpha(t) R^{2}(\alpha(t)$ is a dimensionless scalar) is added to Einstein-Hilbert lagrangian using the approach in [25]. Unlike in $[16,17,18,19,21,22,23,24]$, here $\alpha(t) R^{2}$ is not lagrangian density for DE. Rather DE and dark matter (DM) are induced due to presence of this term in the gravitational action. DE, obtained here, mimics quintessnce. It is unlike the approach in $[16,17,18,19,21,22,23,24]$, where non-linear term of $R$ is used as DE lagrangian.

In $[26,27,28,29]$, it is found that if higher-derivative terms are added to Einstein-Hilbert action, the Ricci scalar also manifests a physical field in addition to its usual role as geometrical field. In the present model, field equations from the modified gravitational action yields Friedmann equations with DED, DMD and radiation density. DE and DM are physical concepts. Spontaneous emergence of these physical terms from gravitational sector shows that these are caused by dual roles of $R$ (as a physical as well as a geometrical field).

It leads to a very interesting cosmology. Here, cosmological scenario begins with power-law inflation for a fraction of a second and universe expands $10^{27}$ times. Later on, radiation dominates and universe decelerates. Around 386 kyr of age, Matter dominates over radiation and deceleration continues. At red-shift $Z_{* *} \simeq 0.54$, a transition takes place. DE re-dominates and decelerating universe gets speeded up. DED, obtained here, falls from a very high value $1.19 \times 10^{75} \mathrm{GeV}^{4}$ at Planck scale to its current value.

The paper is organized as follows. Section 2(a) contains gravitational action and manifestation of curvature scalar as a physical field. Emergence of DE, DM and radiation is discussed in scetion 2(b). Scalar field models for DE have been very popular. Though DE has gravitational 
origin here, still it is interesting to probe scalar field and its corresponding potential giving gravitationally originated DE. This probe is done in section 2(c). Section 3 contains discussion on the resulting cosmological picture. In section 4, it is shown that, actually, our universe is residing in a much larger universe driven by DE only. In the last scetion, results of the paper are summarized.

Natural units $(\hbar=c=1)$ are used here with $\mathrm{GeV}$ as the fundamental unit, where $\hbar$ and $c$ have their usual meaning. In this unit, it is found that $1 \mathrm{GeV}^{-1}=6.58 \times 10^{-25}$ sec.

\section{Dark energy and dark matter caused by dual role}

\section{of the Ricci scalar curvature}

\section{(a) Dual role of the curvature scalar}

Here, investigations begin with the action for higher-derivative gravity

$$
S_{g}=\int d^{4} x \sqrt{-g}\left[\frac{1}{16 \pi G}(R-2 \Lambda(t))+\alpha(t) R^{2}\right],
$$

where $R$ is the Ricci scalar curvature, $G=M_{P}^{-2}\left(M_{P}=10^{19} \mathrm{GeV}\right.$ is the Planck mass) and dimensionless $\alpha$ is a scalar depending on cosmic time $t$. Here, $\Lambda(t)$ is another scalar having dimension (mass) ${ }^{2}$

The action (2.1) yields gravitational field equations

$$
\begin{aligned}
& \frac{1}{16 \pi G}\left[R_{\mu \nu}-\frac{1}{2} g_{\mu \nu}(R-2 \Lambda(t)]+\alpha(t)\left[2 \nabla_{\mu} \nabla_{\nu} R-2 g_{\mu \nu} \square R-\frac{1}{2} g_{\mu \nu} R^{2}+2 R R_{\mu \nu}\right]\right. \\
& \quad+2\left(\nabla_{\mu} \nabla_{\nu} \alpha-g_{\mu \nu} \square \alpha\right) R+4\left(\nabla_{\mu} \alpha \nabla_{\nu} R-g_{\mu \nu} \nabla^{\sigma} \alpha \nabla_{\sigma} R\right)+=0
\end{aligned}
$$

using the condition $\delta S_{g} / \delta g^{\mu \nu}=0$. Here, $\nabla_{\mu}$ denotes covariant derivative and the operator $\square$ is given as

$$
\square=\frac{1}{\sqrt{-g}} \frac{\partial}{\partial x^{\mu}}\left(\sqrt{-g} g^{\mu \nu} \frac{\partial}{\partial x^{\nu}}\right)
$$

with $\mu, \nu=0,1,2,3$ and $g_{\mu \nu}$ as metric tensor components. 
Taking trace of eqs.(2.2), it is obtained that

$$
\square R+m^{2} R+2 \frac{\dot{\alpha}}{\alpha} \dot{R}=\frac{\Lambda(t)}{24 \pi G \alpha(t)},
$$

where

$$
m^{2}=\frac{1}{96 \pi G \alpha(t)}+\frac{\square \alpha}{\alpha}
$$

with $\alpha(t)>0$ to avoid the ghost problem. Here, overdot gives derivative with respect to time $t$.

Eq.(2.4a) is typically of the Klein - Gordon form with the source term $\frac{\Lambda(t)}{24 \pi G \alpha(t)}$. It shows that the Ricci scalar $R$ behaves as a physical field also with (mass) ${ }^{2}$ depending on $G$, in addition to its usual role as a geometrical field [26, 27, 28, 29].

\section{(b) Emergence of dark energy and dark matter}

Experimental evidences support spatially homogeneous flat model of the universe [30]. So, the line-element, giving geometry of the universe, is taken as

$$
d S^{2}=d t^{2}-a^{2}(t)\left[d x^{2}+d y^{2}+d z^{2}\right]
$$

with $a(t)$ as the scale factor.

In the space-time, given by eq.(2.5), eq.(2.4a) is obtained as

$$
\ddot{R}+3 \frac{\dot{a}}{a} \dot{R}+\left(\frac{1}{96 \pi G \alpha(t)}+\frac{\ddot{\alpha}}{\alpha}+3 \frac{\dot{a}}{a} \frac{\dot{\alpha}}{\alpha}\right) R+2 \frac{\dot{\alpha}}{\alpha} \dot{R}+\frac{\Lambda(t)}{24 \pi G \alpha(t)}=0
$$

using eq. (2.4b) for $m^{2}$.

In most of the situations (radiation model, matter-dominated model and dark energy dominated models), we have $a(t)$ as a power-law solution, for which $R$ is obtained as power-law function of $a(t)$. So, it is reasonable to take

$$
R=\frac{A}{a^{n}}
$$

where $n \neq 0$ is a real number and $A$ is a constant. 
Moreover, scalars $\alpha(t)$ and $\Lambda(t)$ are taken as

$$
\alpha(t)=D a^{r}
$$

and

$$
\Lambda(t)=E a^{-p}
$$

with $r \neq 0$ and $p \neq 0$ being real numbers. Here $D$ and $E$ are positive constants. In what follows, we get energy density in the form by taking $\alpha(t)$ and $\Lambda(t)$ in these forms.

$R, \alpha(t)$ and $\Lambda(t)$ (given by eqs.(2.7),(2.8) and (2.9) respectively) satisfy eq.(2.6), if

$$
\frac{\ddot{a}}{a}-[n-r-2]\left(\frac{\dot{a}}{a}\right)^{2}=\frac{a^{-r}}{96 \pi A G[n-r]}-\frac{B a^{-p-r+n}}{48 \pi G[n-r]} .
$$

This equation has first integral

$$
\left(\frac{\dot{a}}{a}\right)^{2}=\frac{C}{a^{2[3-n+r]}}+\frac{a^{-r}}{\pi A G[n-r][3-n+r]}-\frac{B a^{-p-r+n}}{48 \pi G[n-r][-p-n+r+6]},
$$

where $C$ is an integration constant. Moreover, $D$ is absorbed in $A$ as well as $B$ absorbs both $A$ and $D$.

Taking real numbers $n$ and $r$ such that $n-r=3 / 2$, eq.(2.11) looks like

$$
\left(\frac{\dot{a}}{a}\right)^{2}=\frac{C}{a^{3}}+\frac{a^{-r}}{144 \pi A G[3-r]}-\frac{B a^{-p+\frac{3}{2}}}{18 \pi G[-2 p+9]},
$$

The third term on the right hand side (r.h.s.) of eq.(2.12) is positive, if

$$
p>\frac{9}{2} .
$$

So, $p=\frac{11}{2}$ can be taken. Thus eq.(2.12) takes the form

$$
\left(\frac{\dot{a}}{a}\right)^{2}=\frac{C}{a^{3}}+\frac{1}{144 \pi A G[3-r] a^{r}}+\frac{B}{36 \pi G a^{4}} .
$$


This is the Friedmann equation with density

$$
\rho=\frac{3}{8 \pi G}\left[\frac{C}{a^{3}}+\frac{1}{144 \pi A G[3-r] a^{r}}+\frac{B}{36 \pi G a^{4}}\right]
$$

giving dynamics of the universe.

The first term on r.h.s. of eq.(2.15)

$$
\rho_{m}=\frac{3}{8 \pi G} \frac{C}{a^{3}}
$$

is like density of matter emerging from gravity. So, it is termed as dark matter density (DMD). Similarly the second term

$$
\rho_{\mathrm{de}}=\frac{3}{8 \pi G}\left[\frac{1}{144 \pi A G[3-r] a^{r}}\right]
$$

is also inspired by gravity. So it is recognized as dark energy density (DED). The third term on r.h.s. of eq.(2.15)

$$
\rho_{r}=\frac{3}{8 \pi G}\left[\frac{B}{36 \pi G a^{4}}\right]
$$

has the form of radiation density, which is obtained taking $\Lambda(t) \propto$ $a^{-11 / 2}(t)$. In [10], $\Lambda(t) \propto a^{-p}(t)$ is taken to explain DE. Here this term gives radiation and DE is explained by another term mentioned above.

The conservation equation for $\mathrm{DE}$ is given as

$$
\dot{\rho}_{\mathrm{de}}+3 \frac{\dot{a}}{a}\left(1+\mathrm{w}_{\mathrm{de}}\right) \rho_{\mathrm{de}}=0
$$

where equation of state parameter $(\mathrm{EOSP}) \mathrm{w}_{\mathrm{de}}=p_{\mathrm{de}} / \rho_{\mathrm{de}}$ with $p_{\mathrm{de}}\left(\rho_{\mathrm{de}}\right)$ being pressure(density) for DE.

Connecting eqs.(2.17) and (2.19), it is obtained as

$$
r=3\left(1+\mathrm{w}_{\mathrm{de}}\right)
$$

According to WMAP $[31, ?]$, current values of $\rho_{m}, \rho_{\mathrm{de}}$ and $\rho_{r}$ are $\rho_{m}^{0}=0.27 \rho_{\mathrm{cr}}^{0}, \rho_{\mathrm{de}}^{0}=0.73 \rho_{\mathrm{cr}}^{0}$ and $\rho_{r}^{0}=5 \times 10^{-5} \rho_{\mathrm{cr}}^{0}$, where $\rho_{\mathrm{cr}}^{0}=3 H_{0}^{2} / 8 \pi G, H_{0}=$ $100 \mathrm{hkm} / \mathrm{Mpcsec}=2.33 \times 0.68 \times 10^{-42} \mathrm{GeV}$ using $\mathrm{h}=0.68($ a value having the maximum likelihood ). 
These observational values are used to determine constants in eqs.(2.16),(2.17) and (2.18). As a result,

$$
\begin{gathered}
\rho_{m}=0.27 \rho_{\mathrm{cr}}^{0}\left(\frac{a_{0}}{a}\right)^{3}, \\
\rho_{\mathrm{de}}=0.73 \rho_{\mathrm{cr}}^{0}\left(\frac{a_{0}}{a}\right)^{3\left(1+\mathrm{w}_{\mathrm{de}}\right)},
\end{gathered}
$$

and

$$
\rho_{r}=5 \times 10^{-5} \rho_{\mathrm{cr}}^{0}\left(\frac{a_{0}}{a}\right)^{4},
$$

Now Friedmann equation (2.14) takes the form

$$
\left(\frac{\dot{a}}{a}\right)^{2}=H_{0}^{2}\left[0.27\left(\frac{a_{0}}{a}\right)^{3}+0.73\left(\frac{a_{0}}{a}\right)^{3\left(1+\mathrm{w}_{\mathrm{de}}\right)}+5 \times 10^{-5}\left(\frac{a_{0}}{a}\right)^{4}\right] .
$$

Terms, on r.h.s. of eq.(2.24), are physical concepts, whereas term on the 1.h.s. is geometrical. All these terms arise from the gravitational action (2.1). It is due to manifestation of $R$ as a physical field [29].

\section{(c) Scalar field for dark energy}

Here, DE is obtained from higher-derivative gravity. But, in nongravitational models, it is caused by some exotic matter given by a scalar field (quintessence, tachyon and phantom). Though, we have a gravitational origin of DE here, it is natural to probe a scalar $\phi$ (which is not a field for an exotic matter) giving $\rho_{\text {de }}$ obtained above. With $V(\phi)$ as potential, $\phi$ obeys the equation

$$
\ddot{\phi}+3 \frac{\dot{a}}{a} \dot{\phi}+V^{\prime}(\phi)=0
$$

and has mass dimension equal to 1 . Here, $V^{\prime}(\phi)=d V / d \phi$. DE density and pressure, in terms of $\phi$, are given as

$$
\rho_{\mathrm{de}}=\frac{1}{2} \dot{\phi}^{2}+V(\phi)
$$

and

$$
p_{\mathrm{de}}=\frac{1}{2} \dot{\phi}^{2}-V(\phi)
$$


Eqs.(2.26a) and (2.26b) yield

$$
\rho_{\mathrm{de}}=\frac{\dot{\phi}^{2}}{1+\mathrm{w}_{\mathrm{de}}}
$$

and

$$
V(\phi)=\frac{1}{2}\left(1-\mathrm{w}_{\mathrm{de}}\right) \rho_{\mathrm{de}}
$$

using $p_{\mathrm{de}}=\mathrm{w}_{\mathrm{de}} \rho_{\mathrm{de}}$. Now, from eqs. $(2.21 \mathrm{a})$ and (2.27a)

$$
\dot{\phi}^{2}=\frac{2.19}{8 \pi G}\left(1+\mathrm{w}_{\mathrm{de}}\right) H_{0}^{2}\left(\frac{a_{0}}{a}\right)^{3\left(1+\mathrm{w}_{\mathrm{de}}\right)}
$$

and

$$
V(\phi)=\frac{2.19}{8 \pi G}\left(1-\mathrm{w}_{\mathrm{de}}\right) H_{0}^{2}\left(\frac{a_{0}}{a}\right)^{3\left(1+\mathrm{w}_{\mathrm{de}}\right)}
$$

\section{Cosmic evolution}

In what follows, cosmic evolution passes through four different phases.

\section{Phase 1. Power - law inflation}

According to the present cosmological picture, in the beginning, the universe contains DE only. So, Friedmann equation (2.24) looks like

$$
\left(\frac{\dot{a}}{a}\right)^{2}=0.73 H_{0}^{2}\left(\frac{a_{0}}{a}\right)^{3\left(1+\mathrm{w}_{\mathrm{de}}\right)}
$$

which is integrated to

$$
a(t)=a(0)\left[1+\sqrt{0.73} H_{0}\left\{\frac{3\left(1+\mathrm{w}_{\mathrm{de}}\right)}{2}\right\}\left(\frac{a_{0}}{a}\right)^{3\left(1+\mathrm{w}_{\mathrm{de}}\right) / 2} t\right]^{2 / 3\left(1+\mathrm{w}_{\mathrm{de}}\right)},
$$

where $a(0)=a(t=0)$.

As $\mathrm{w}_{\mathrm{de}}<-1 / 3,3\left(1+\mathrm{w}_{\mathrm{de}}\right)>2$. Hence $a(t)$, given by eq.(3.2), exhibits power-law inflation as $\ddot{a}(t)>0$. Moreover, as $1+3 \mathrm{w}_{\mathrm{de}}<0$, SEC is violated. It shows bounce of the universe at $t=0$, so $a(0) \neq 0[29,33]$

In terms of $\phi$ (given above in scetion 2(c)), eq.(3.1) is written as

$$
\frac{3 H^{2}}{8 \pi G}=\rho_{\mathrm{de}}=\frac{1}{2} \dot{\phi}^{2}+V(\phi)=\frac{\dot{\phi}^{2}}{1+\mathrm{w}_{\mathrm{de}}}
$$


using eqs.(2.26a) and (2.27a). Here $H=\frac{\dot{a}}{a}$. Eq.(3.3) yields

$$
\dot{\phi}=H \sqrt{\frac{3\left(1+\mathrm{w}_{\mathrm{de}}\right)}{8 \pi G}}
$$

and

$$
\frac{3 H H^{\prime}}{4 \pi G}=\frac{1}{2} \frac{d \dot{\phi}^{2}}{d \phi}+V^{\prime}(\phi)=-3 H \dot{\phi}
$$

using eq.(2.25). Here, $H^{\prime}=d H / d \phi$. Further, eqs.(3.4a) and (3.4b) imply

$$
\frac{H^{\prime}}{4 \pi G}=-H \sqrt{\frac{3\left(1+\mathrm{w}_{\mathrm{de}}\right)}{8 \pi G}}
$$

giving the slow-roll parameter

$$
\epsilon=\frac{1}{4 \pi G}\left(\frac{H^{\prime}}{H}\right)^{2}=\frac{3\left(1+\mathrm{w}_{\mathrm{de}}\right)}{2}
$$

Experimental results restrict $\epsilon \lesssim 0.05$ [34]. So, taking $\epsilon \simeq 0.05$, eq.(3.6) yields

$$
\frac{3\left(1+\mathrm{w}_{\mathrm{de}}\right)}{2}=\frac{1}{20}
$$

and

$$
\mathrm{w}_{\mathrm{de}}=-\frac{29}{30} .
$$

It shows that DE, obtained here, mimics quintessence as $\mathrm{w}_{\mathrm{de}}>-1$.

Observed perturbation amplitude, on large scale, before the end of inflation is given by [34]

$$
\frac{8 V_{*}}{3 M_{P}^{4} \epsilon} \simeq 2.6 \times 10^{-9}
$$

where $V_{*}$ is the potential at the end of inflation. The maximum number of e-fold for the inflation [34]is

$$
N^{\max }=63.3+0.25 \ln \epsilon \simeq 62.55 .
$$

If $a_{*}$ is the scale factor at the end of inflation

$$
\frac{a_{*}}{a(0)}=e^{62.55}=1.46 \times 10^{27} .
$$


Connecting eqs.(2.27b) and (3.8), at the end of inflation, DED is obtained as

$$
\rho_{\mathrm{de}}\left(t_{*}\right)=\frac{7.8 \times 10^{-9} M_{P}^{4} \epsilon}{4\left(1-\mathrm{w}_{\mathrm{de}}\right)}=4.96 \times 10^{65} \mathrm{GeV}^{4}
$$

using $\epsilon$ and $\mathrm{w}_{\mathrm{de}}$ from eqs.(3.7a,b).

Eq.(2.22) yields

$$
\rho_{\mathrm{de}}(0)=\rho_{\mathrm{de}}\left(t_{*}\right)\left[\frac{a_{*}}{a(0)}\right]^{3\left(1+\mathrm{w}_{\mathrm{de}}\right)}=2.58 \times 10^{68} \mathrm{GeV}^{4}
$$

using eqs.(3.7a) and (3.10). From eqs.(2.22) and (3.12), it is obtained that

$$
\left[\frac{a_{0}}{a(0)}\right]^{3\left(1+\mathrm{w}_{\mathrm{de}}\right)}=1.18 \times 10^{115} \mathrm{GeV}^{4}
$$

yielding an exceptionally large value of $a_{0} / a(0)$ as

$$
\frac{a_{0}}{a(0)}=5.23 \times 10^{1150}
$$

For the time being, we postpone a discussion on this result to the next section.

Using the results (3.7a) and (3.13a), eq.(3.2) is obtained as

$$
a(t)=a(0)\left[1+1.52 \times 10^{14} t\right]^{20} .
$$

Incorporation of the result (3.10) for $a_{*}$ in eq.(3.14), the inflation period $t_{*}$ is evaluated as

$$
t_{*} \simeq 1.44 \times 10^{-13} \mathrm{GeV}^{-1}=9.44 \times 10^{-38} \mathrm{sec} .
$$

Integrating eq.(3.4a), we obtain

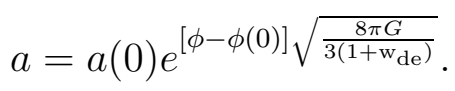

Connecting eqs.(2.28b) and (3.16)

$$
\begin{aligned}
V(\phi) & =\frac{2.19}{16 \pi G}\left(1-\mathrm{w}_{\mathrm{de}}\right) H_{0}^{2}\left[\frac{a_{0}}{a(0)}\right]^{3\left(1+\mathrm{w}_{\mathrm{de}}\right)} e^{-[\phi-\phi(0)] \sqrt{\frac{4 \pi G}{5}}} \\
& =7.12 \times 10^{62} e^{-[\phi-\phi(0)] \sqrt{\frac{4 \pi G}{5}}}
\end{aligned}
$$


using eqs.(3.7b) and (3.13a). At the end of inflation,

$$
\phi_{*}-\phi(0)=\sqrt{\frac{3\left(1+\mathrm{w}_{\mathrm{de}}\right)}{8 \pi G}} \ln \left(\frac{a_{*}}{a(0)}\right)=3.945 \times 10^{19} \mathrm{GeV}
$$

connecting eqs.(3.7b), (3.10) and (3.16). Here $\phi_{*}=\phi\left(t_{*}\right)$.

\section{Phase 2. Radiation-dominated phase}

It is found above that, during phase 1, the universe inflates for a very small fraction of a second and eqs.(3.11) and (3.12) show that $\rho_{\mathrm{de}}$ falls by 3 orders upto the end of inflation. The density for released DE is obtained as

$$
\rho_{\mathrm{de}(\text { released })}=\rho_{\mathrm{de}}(0)-\rho_{\mathrm{de}}\left(t_{*}\right)=2.56 \times 10^{68} \mathrm{GeV}^{4}
$$

using eqs.(3.11) and (3.12).

It is proposed that the released DE decays to emission of radiation and elementary particles in thermal equillibrium with radiation, heralding the usual big-bang cosmology. It is parallel to the idea introduced by Bronstein in 1933 [6]. Here, the emitted radiation is recognized as microwave background radiation. So,

$$
\rho_{\text {de(released })}=\rho_{r}\left(t_{*}\right)=\frac{\pi^{2}}{15} T_{*}^{4} .
$$

Initial temperature for this radiation is obtained as

$$
T_{*}=1.4 \times 10^{17} \mathrm{GeV} .
$$

from eqs.(3.19) and (3.20). Moreover, it is obtained that

$$
\rho_{r}\left(t_{*}\right)>\rho_{\mathrm{de}}\left(t_{*}\right)
$$

Also matter remains in thermal equillibrium with radiation till decoupling time. 
Hence, this phase of evolution is radiation-dominated. Now, during the period $t_{*}$ and decoupling time $t_{D}$ Friedmann equation (2.24) is obtained as

$$
\left(\frac{\dot{a}}{a}\right)^{2}=5 \times 10^{-5} H_{0}^{2}\left(\frac{a_{0}}{a}\right)^{4}
$$

which integrates to

$$
a(t)=a_{*}\left[1+2 \beta\left(t-t_{*}\right)\right]^{1 / 2}
$$

with

$$
\beta=\sqrt{5 \times 10^{-5}} H_{0}\left(\frac{a_{0}}{a_{*}}\right)^{2} .
$$

In terms of $\phi$ eq.(3.22) can be re-written as

$$
\dot{\phi}^{2}\left(\frac{1}{a} \frac{d a}{d \phi}\right)^{2}=5 \times 10^{-5} H_{0}^{2}\left(\frac{a_{0}}{a}\right)^{4} .
$$

So,

$$
\left(\frac{1}{a} \frac{d a}{d \phi}\right)^{2}=\frac{5 \times 10^{-5} H_{0}^{2}}{\left(1+\mathrm{w}_{\mathrm{de}}\right) \rho_{\mathrm{de}}}\left(\frac{a_{0}}{a}\right)^{4}=1.77 \times 10^{-42}\left(\frac{a_{0}}{a}\right)^{39 / 10}
$$

using eq.(2.27a) and $\mathrm{w}_{\mathrm{de}}=-29 / 30$.

Eq.(3.24) is integrated to

$$
a(t)=a_{*}\left[1+2.56 \times 10^{-20}\left(\frac{a_{0}}{a_{*}}\right)^{39 / 20}\left(\phi-\phi_{*}\right)\right]^{20 / 39} .
$$

Connecting eqs.(2.28b) and (3.25), it is obtained that

$$
V(\phi)=4.3 \times 10^{-47}\left(\frac{a_{0}}{a_{*}}\right)^{1 / 10}\left[1+2.56 \times 10^{-20}\left(\frac{a_{0}}{a_{*}}\right)^{39 / 20}\left(\phi-\phi_{*}\right)\right]^{-2 / 39} .
$$

\section{$\underline{\text { Phase 3. Matter-dominated phase }}$}

Matter dominates over radiation, $\rho_{m}>\rho_{r}$, when

$$
0.27>5 \times 10^{-5}\left(\frac{a_{0}}{a}\right)
$$

using $\rho_{m}$ and $\rho_{r}$ from eqs.(2.21) and (2.23) respectively. 
Defining red-shift as

$$
1+z=\frac{a_{0}}{a}
$$

the inequality (3.27) yields that matter dominates over radiation at

$$
z<5400 \text {. }
$$

It is consistent with WMAP [31], which gives that matter decouples from radiation at time $t_{d}=386 \mathrm{kyr}=1.85 \times 10^{37} \mathrm{GeV}^{-1}$ and red-shift

$$
Z_{d}=\frac{a_{0}}{a_{d}}-1=1089
$$

So, we take the red-shift $Z_{d}=1089$, when matter dominates over radiation. In this case, the Friedmann equation (2.24) reduces to

$$
\left(\frac{\dot{a}}{a}\right)^{2}=0.27 H_{0}^{2}\left(\frac{a_{0}}{a}\right)^{3}
$$

which integrates to

$$
a(t)=a_{d}\left[1+2 \gamma\left(t-t_{d}\right)\right]^{2 / 3},
$$

with

$$
\gamma=\sqrt{0.27} H_{0}\left(\frac{a_{0}}{a_{d}}\right)^{3 / 2}=2.96 \times 10^{-38} \mathrm{GeV}
$$

using eq.(3.29).

In terms of $\phi$ eq.(3.30) can be re-written as

$$
\dot{\phi}^{2}\left(\frac{1}{a} \frac{d a}{d \phi}\right)^{2}=0.27 H_{0}^{2}\left(\frac{a_{0}}{a}\right)^{3} .
$$

So,

$$
\left(\frac{1}{a} \frac{d a}{d \phi}\right)^{2}=\frac{0.27 H_{0}^{2}}{\left(1+\mathrm{w}_{\mathrm{de}}\right) \rho_{\mathrm{de}}}\left(\frac{a_{0}}{a}\right)^{3}=9.3 \times 10^{-37}\left(\frac{a_{0}}{a}\right)^{29 / 10}
$$

using eq. (2.27a) and $\mathrm{w}_{\mathrm{de}}=-29 / 30$.

Eq.(3.32) is integrated to

$$
a(t)=a_{d}\left[1+3.54 \times 10^{-14}\left(\phi-\phi_{d}\right)\right]^{20 / 29} .
$$


Connecting eqs.(2.28b) and (3.33), it is obtained that

$$
V(\phi)=4.33 \times 10^{-47}\left[1+5.5 \times 10^{-23}\left(\phi-\phi_{d}\right)\right]^{-2 / 29} .
$$

Further, according to measurements [35, 37], gamma-ray bursts (GRBs) are found in sub-luminous star forming host of galaxies. Collapsar model suggests that these are likely produced in core-collapse explosions of a class of massive stars giving rise to relativistic jets. It shows existence of massive stars, when GRBs are produced. GRBs are found at high red-shift $z \sim 1.6$ [36]. The high red-shift $z_{B}=6.1_{-0.12}^{+0.36}$ for recently observed GRB 050904 [37] beats all previous records. Using eq.(3.31) it is obtained that

$$
1+Z_{B}=\frac{a_{0}}{a\left(t_{B}\right)}=\frac{a_{0}}{a_{d}}\left[1+4.44 \times 10^{-38}\left(t_{B}-t_{d}\right)\right]^{-2 / 3}
$$

Using eq.(3.29) for $a_{0} / a_{d}$ and $z_{B}$ given above, time for GRB $t_{B}$ is evaluated from 822 Myr to 918 Myr after big-bang showing preesence of massive stars by this time.

\section{Phase 4. Dominanace of dark energy in the late universe}

Dominance of DE over matter $\left(\rho_{\mathrm{de}}>\rho_{m}\right)$ is possible when

$$
0.27<0.73\left(\frac{a_{0}}{a}\right)^{-29 / 10}
$$

using $\rho_{m}$ and $\rho_{r}$ from eqs.(2.21) and (2.22) respectively.

The inequality (3.36) shows that dominance of DE, in the late universe, is possible, when

$$
\frac{a_{0}}{a}=1+z<1.409
$$

using $\mathrm{w}_{\mathrm{de}}=-29 / 30$ obtained above.

So, we can take red-shift for the transition from $\rho_{\text {de }}<\rho_{m}$ to $\rho_{\text {de }}>\rho_{m}$ as

$$
z_{* *}=0.4
$$


safely. This is the red-shift at which DE dominates over matter. As cosmic acceleration is caused by dominance of DE. $z_{* *}$ is the red-shift for transition from deceleration to acceleration.

Interestingly, it falls in the range of red-shift $z=0.46 \pm 0.13$ for this transition, given by 16 Type supernova (SNe Ia) [5].

In this case, the Friedmann equation (2.24) reduces to

$$
\left(\frac{\dot{a}}{a}\right)^{2}=0.73 H_{0}^{2}\left(\frac{a_{0}}{a}\right)^{1 / 10}
$$

which integrates to

$$
a(t)=a_{* *}\left[1+\omega\left(t-t_{* *}\right)\right]^{20},
$$

with

$$
\omega=0.04 H_{0}\left(\frac{a_{0}}{a_{* *}}\right)^{1 / 20}\left(\frac{a_{0}}{a_{* *}}\right)^{1 / 20}=6.88 \times 10^{-44} \mathrm{GeV}
$$

and $a_{* *}=a\left(t_{* *}\right)$. Here $t_{* *}$ is the transition time. At the present time $t_{0}=13.7 \mathrm{Gyr}=6.6 \times 10^{41} \mathrm{GeV}^{-1}$ we have

$$
1+z_{* *}=\frac{a_{0}}{a_{* *}}=\left[1+6.88 \times 10^{-44}\left(t_{0}-t_{* *}\right)\right]^{20} .
$$

Connecting eqs.(3.38) and (3.40), it is evaluated that

$$
t_{* *}=4.13 \times 10^{41} \mathrm{GeV}^{-1}=8.58 \mathrm{Gyr} .
$$

Eq.(3.40) shows accelerated expansion beginning at $t_{* *}$. So, 8.58Gyr is the time for transition from deceleration to acceleration in the late universe.

In terms of $\phi$, eq.(3.3) has the solution for this case as

$$
a=a_{* *} e^{\left[4 \sqrt{5 \pi G}\left(\phi-\phi_{* *}\right)\right]},
$$

where $\phi_{* *}=\phi\left(t_{* *}\right)$.

Connecting eqs.(2.28b) and (3.42), it is obtained that

$$
V(\phi)=2.22 \times 10^{37} e^{\left[-\sqrt{4 \pi G / 5}\left(\phi-\phi_{* *}\right)\right]} .
$$




\section{Universe driven by dark energy}

In this section, we probe what could be the shape of the universe driven purely by DE from beginning upto the present time. In this case, cosmic dynamics is given by eq.(3.1) yielding the scale factor (3.2). From $a(t)$, given by eq.(3.2), the rate of expansion rate is obtained as

$$
\tilde{H}=\frac{\sqrt{0.73} H_{0}\left(\frac{a_{0}}{a(0)}\right)^{1 / 20}}{\left[1+\frac{1}{20} \sqrt{0.73} H_{0}\left(\frac{a_{0}}{a(0)}\right)^{1 / 20} t\right]}
$$

with $\mathrm{w}_{\mathrm{de}}=-29 / 30$ obtained above. Using eqs.(3.13a,b) in eq.(4.1)

$$
\tilde{H} \simeq \frac{20}{t} .
$$

At $t=t_{0}=13.7 G y r=6.6 \times 10^{41} \mathrm{GeV}^{-1}$, eq.(4.2) yields

$$
\tilde{H}_{0}=3.05 \times 10^{-42} \mathrm{GeV}=19.25 H_{0} .
$$

It means that present Hubble's rate $H_{0} \rightarrow \tilde{H}_{0}=19.25 H_{0}$ in the universe (driven by DE only). It happens due to highly accelerating expansion driven by DE having density (2.22). The equation (4.3) shows that, in this situation, $H$ is rescaled as $19.25 H$. With this rescaling, eq.(3.1) looks like

$$
\left(\frac{\dot{\tilde{a}}}{\tilde{a}}\right)^{2}=\left(19.25 \frac{\dot{a}}{a}\right)^{2}=0.73 \tilde{H}_{0}^{2}\left(\frac{\tilde{a}_{0}}{\tilde{a}}\right)^{1 / 10}
$$

and eq.(3.2) takes the form

$$
\tilde{a}(t)=\tilde{a}(0)\left[1+\frac{1}{20} \sqrt{0.73} H_{0}\left(\frac{a_{0}}{a(0)}\right)^{1 / 20} t\right]^{20},
$$

which yields

$$
\frac{\tilde{a}_{0}}{\tilde{a}(0)} \simeq \frac{a_{0}}{a(0)}=5.23 \times 10^{1150} .
$$

This result is found for an universe, which is not being observed. We call it actual universe Now we come to our observable universe.

\section{$\underline{\text { Observable universe }}$}


As discussed in the preceding section, universe passes through 4 different phases ( power-law inflation, radiation-dominated, matterdominated and DE dominated phase). We call it observable universe. According to analysis for observable universe, eq.(3.10) gives the ratio

$$
\left(\frac{a_{*}}{a(0)}\right)_{\text {obs. }}=1.46 \times 10^{27}
$$

At $t=t_{*}\left(t_{*}\right.$ being the time for end of inflation), eq.(2.23) yields

$$
\rho_{r}\left(t_{*}\right)=\frac{15 \times 10^{-5}}{8 p i G} H_{0}^{2}\left(\frac{a_{0}}{a_{*}}\right)_{\text {obs. }}^{4} .
$$

Connecting eqs.(3.19), (3.20) and (4.8), we obtain that

$$
\left(\frac{a_{0}}{a_{*}}\right)_{\text {obs. }}=6.43 \times 10^{29}
$$

From eqs.(4.7) and (4.9)

$$
\left(\frac{a_{0}}{a(0)}\right)_{\text {obs. }}=9.39 \times 10^{56} .
$$

So, from eqs.(4.6)and (4.10), it is obtained that

$$
\left(\frac{a_{0}}{a(0)}\right)_{\text {actual }}=5.23 \times 10^{1150}>>\left(\frac{a_{0}}{a(0)}\right)_{\mathrm{obs}}
$$

Thus, the result (4.11) leads to a very interesting conclusion that actually, our observable universe resides in an extremely large universe driven by DE only. As $H_{0} \rightarrow \tilde{H}_{0}=19.25 H_{0}$

$$
\rho_{\text {de(actual })}=370.56 \rho_{\text {de(obs. })} .
$$

It means that only $\sim \frac{1}{371}$ of $\mathrm{DE}$ is available in our observable universe.

The reason for this scenario is given as follows. Due to the reverse gravity effect of DE, the actual universe speeds up very fast, whereas the observable universe driven by radiation and subsequently by matter decelerats for the major portion of its age. So, the observable universe is concentrated in a very small part of the actual universe. Interestingly, this conclusion is based on observed physical parameters and results 
(derived using observations) that DE density falls with the expansion of the universe by 115 orders upto the present epoch.

\section{Summary}

It is interesting to see that, in the present model, DE and DM emerge spontaneously due to manifestation of the Ricci scalar curvature $R$ as a physical field too. It is obtained from higher-derivative gravitational action, which stems from adding a non-linear term $\alpha(t) R^{2}$ to EinsteinHilbert term. Here, $\alpha$ is a dimensionless scalar varying with scale factor. In $[16,17,18,19,20,21,22,23,24]$, theory with non-linear term of $R$ is called modified gravity, where coupling term $\alpha$ is a constant. In these papers on modified gravity, the non-linear term of $R$ stands for the lagrangian density of $\mathrm{DE}$ and it is added to explain late acceleration in the universe. In [19], Nojiri and Odintsov have taken non-linear $a R^{m}+b R^{-n}$ with the motive to have inflation in the early universe and late accelartion. In contrast to all these works, in the present paper, nothing is taken a priori to get DE. Here, DE, DM and radiation are induced by modified gravity with non-linear term $\alpha(t) R^{2}$. The resulting cosmological scenario is given as follows. In the beginning, our pbservable universe inflates for $\sim 10^{-37}$ sec. DE, released during this period, decays to emmission of CMB radiation and light elementary particles. This event heralds usual big-bang expansion with an end of inflation as emitted radiation dominates over DE by the time $10^{-37}$ sec. After this epoch, universe decelerates for a long time, driven by radiation and subsequently by matter. Around the age 8.58Gyr of the universe, a transition takes place and DE re-dominates over matter, giving a cosmic jerk. As a result, decelerating universe gets speeded 
up [25]. DE density, obtained here, falls from $2.58 \times 10^{68} \mathrm{GeV}^{4}$ to its current value $2.18 \times 10^{-47} \mathrm{GeV}^{4}$.

In non-gravitational field theoretic models of DE, a scalar field $\phi(t)$ (quintessence, k-essence, tachyon and phantom ) are taken as DE source. In contrast to this approach, here, DE is obtained from gravitational sector and $\phi(t)$ is probed for it. Thus, here, $\phi(t)$ has a geometrical origin, whereas origin of quintessence, k-essence and phantom is not known (except tachyon having stringy origin). The potential $V(\phi)$, for DE obtained here, is tabulated below in different situations.

\section{$\underline{\text { Table no.1 }}$}

Potential $V(\phi)$ during different phases of the universe

\begin{tabular}{lll}
\hline$V(\phi)$ & $\begin{array}{l}\text { Dominance in } \\
\text { the universe }\end{array}$ & $\begin{array}{l}\text { Nature of } \\
\text { expansion }\end{array}$ \\
\hline $7.12 \times 10^{62} e^{-[\phi-\phi(0)] \sqrt{\frac{4 \pi G}{5}}}$ & DE & acceleration \\
$4.3 \times 10^{-47}\left(\frac{a_{0}}{a_{*}}\right)^{1 / 10}[1+$ & radiation & deceleration \\
$\left.2.56 \times 10^{-20}\left(\frac{a_{0}}{a_{*}}\right)^{39 / 20}\left(\phi-\phi_{*}\right)\right]^{-2 / 39}$ & & \\
$4.33 \times 10^{-47}[1+$ & $\mathrm{DM}$ & deceleration \\
$\left.5.5 \times 10^{-23}\left(\phi-\phi_{d}\right)\right]^{-2 / 29}$ & & \\
$2.22 \times 10^{37} e^{\left[-\sqrt{4 \pi G / 5}\left(\phi-\phi_{* *}\right)\right]}$ & $\mathrm{DE}$ & acceleration \\
\hline
\end{tabular}

In $[38,39]$, considering $V(\phi) \sim e^{-c \phi}$ ( $c$ being a constant) cosmic acceleration is derived in flat homogeneous model of the universe. Here the result is opposite. In the present paper, it is obtained that when universe accelerates, potential falls exponentially and it falls as powerlaw in case of deceleration. 
Moreover it is found that our observable universe is actually residing in a largger universe driven by DE, having equation of state parameter $\mathrm{w}_{\mathrm{de}}=-29 / 30$.

\section{REFERENCES}

[1] S. J. Perlmutter et al., Astrophys. J. 517,(1999)565; astro-ph/9812133.

[2] A. G. Riess et al., Astron. J. 116, (1998) 1009; astro-ph/9805201; J.L. Tonry et al., astro-ph/0305008; P. Garnavich et al, Astrophys. J. Lett. 493 (1998) L53; B.P.Schmidt et al, ibid 507 (1998) 46.

[3] N.A.Bahcall et al, Science, 284 (1991) 1481.

[4] D. N. Spergel et al, Astrophys J. Suppl. 148 (2003)175[ astro-ph/0302209].

[5] A. G. Riess et al, Astrophys. J. 607, (2004) 665 [ astro-ph/0402512].

[6] B. Ratra and P. J. E. Peebles, Rev. Mod.Phys. 75, (2003)559, astroph/0207347; Phys.Rev. D 37, (1988)3406; C. Wetterich, Nucl. Phys. B 302, (1988)668; J. Frieman, C.T. Hill, A. Stebbins and I. Waga, Phys. Rev. Lett. 75, (1995)2077; P. G. Ferreira and M. Joyce, Phys.Rev. D 58, (1998)023503; I. Zlatev, L. Wang and P. J. Steinhardt, Phys. Rev. Lett. 82, (1999)896; P. Brax and J. Martin, Phys.Rev. D 61, (2000)103502; L. A. Ureña-López and T. Matos Phys.Rev. D 62, (2000) 081302(R); T. Barrriro, E. J. Copeland and N.J. Nunes, Phys.Rev. D 61, (2000)127301; A. Albrecht and C. Skordis, Phys. Rev. Lett. D 84, (2000)2076; V. B. Johri, Phys.Rev. D 63, (2001)103504; J. P. Kneller and L. E. Strigari, astro-ph/0302167; F. Rossati, hep-ph/0302159; V. Sahni, M. Sami and T. Souradeep, Phys. Rev. D 65, (2002)023518; M. Sami, N. Dadhich and Tetsuya Shiromizu, hep-th/0304187.

[7] R.R. Caldwell, Phys. Lett. B 545 (2002) 23; R.R. Caldwell, M. Kamionkowski and N.N. Weinberg, Phys. Rev. Lett. 91 (2003) 071301; B. McInnes, JHEP, 08 (2002) 029; hep-th/01120066; Pedro F. González-Díaz, Phys. Rev.D68 (2003) 021303(R); V.K.Onemli et al, Class. Quan. Grav. 19 (2002) 4607 (grqc/0204065); Phys.Rev. D 70 (2004) 107301 (gr-qc/0406098);Class. Quan. Grav. 22 (2005) 59 (gr-qc/0408080) ; V. Sahni \& Yu.V.Shtanov, JCAP 0311 (2003) 014; astro-ph/0202346 ; G.Calcagni, Phys. Rev.D69 (2004) 103508 ; V. Sahni, astro-ph/0502032; S. Noriji and S. D. Odintsov ,Phys.Lett. B 62 (2003) 147 [hep-th/03031147]; E. Elizalde, S. Noriji and S. D. Odintsov ,Phys. Rev.D70(2004) 043539 [hep-th/0405034]; V.B.Johri, Phys.Rev.D 63 (2001) 103504; Class. Quan. Grav. 19 (2002) 5959; Pramanna 59 (2002)1; Phys.Rev. D 70 (2004) 041303 (R) [astro-ph/0311293], S.K.Srivastava; Phys. Lett. B 619 (2005) 1 [astro-ph/0407048], P. Wu and H. Yu; Nucl. Phys.B 727 (2005) 355 [astro-ph/0407424].

[8] S.K.Srivastava; Phys. Lett. B 619 (2005) 1 [astro-ph/0407048].

[9] J.M.Overduin and F.I. Cooperstock, Phys. Rev. D 58 (1998) 043506.

[10] V. Sahni and A. A. Starobinsky, Int. J. Mod. Phys. D 9 (2000) 373 and references therein.

[11] R. Jackiw, physics/0010042.

[12] O. Bertolami et al, Mon. Not.R.Astron.Soc. 353 (2004) 329 [astroph/0402387]. 
[13] M. C. Bento, O. Bertolami, A.A.Sen, Phys. Rev. D 66 (2002)043507 [grqc/0202064]; N. Bilic, G.B.Tupper and R. Viollier, Phys. Lett. B 535 (2002) 17; J.S. Fabris, S.V.Goncalves and P.E. de Soyza, astro-ph/0207430; V. Gorini, A. Kamenshchik and U. Moschella, Phys. Rev. D 67 (2003) 063509 [astroph/0210476]; C. Avelino, L.M.G. Beca, J.P.M. de Carvalho, C.J.A.P. Martins and P. Pinto Phys. Rev. D 67 (2003) 023511 [astr0-ph/0208528].

[14] C. Armendariz-Picon, T. Damour and V. Mukhanov, Phys. Lett. B 458, (1999) 209; T. Chiba, T. Okabe and M. Yamaguchi, Phys. Rev. D 62, (2000)023511.

[15] A. Sen, J. High Energy Phys. 04, (2002)048; 07, (2002)065; Mod. Phys. Lett. A 17, (2002)1799; M. R. Garousi, Nucl. Phys. B 584, (2000)284;J. High Energy Phys. 04, (2003)027; E.A. Bergshoeff et al., J. High Energy Phys. 05, (2000)009; G. W. Gibbons, Phys. Lett. B 537, (2002)1; Parampreet Singh, M. Sami and Naresh Dadhich,Phys.Rev. D 68, (2003)023522 and references therein; A. Mazumdar, S. Panda and A. Perez-Lorenzana, Nucl. Phys. B 584, (2001) 284; S.K.Srivastava, gr-qc/040974.

[16] S.Capozziello, Int.J.Mod.Phys.D 11(2002)483; S.Capozziello, S.Carloni, A.Troisi, astro-ph/0303041.

[17] S.M.Caroll, V.Duvvuri, M.Trodden, M.S.Turner, Phys.Rev. D 70(2004)043528, astro-ph/0306438.

[18] A.D.Dolgov, M.Kawasaki, Phys.Lett.B 573 (2003)1 ; astro-ph/0307285; M.E.Soussa, R.P.Woodard, Gen. Relativ. Gravit. 36 (2004) 855, astroph/0308114; S. Nojiri and S.D.Odintsov, Phys.Lett.A 19(2004)627; hepth/0310045.

[19] S. Nojiri and S.D.Odintsov, Phys.Rev. D 68(2003)123512, hep-th/0307228; M.C.B.Abdalaa, S. Nojiri and S.D.Odintsov, Class. Quan. Grav. 22 (2005) L35, hep-th/0409117.

[20] S. Nojiri and S.D.Odintsov, Gen. Relativ. Gravit. 36 (2004) 1765, hepth/0308176; X.Meng and P.Wang, Phys.Lett.B 584 (2004)1

[21] D. Easson, F.Schuller, M.Trodden, M.Wohlfarth, Phys.Rev. D $\mathbf{7 2}(2005) 043504$, astro-ph/0506392

[22] S.M.Caroll, A.De Felice, V.Duvvuri, D.Easson, M.Trodden, M.S.Turner, Phys.Rev. D 71(2005)063515; G.Allemandi, A.Borowiec, M.Francaviglia, Phys.Rev. D 70(2005)103503.

[23] S..Capozziello, V.Cardone ,A.Troisi, Phys.Rev. D 71(2005)043503; S.Das, N.Banerjee, N.Dadhich, astro-ph/0505096; T.Multamaki, I.Vilja, astro$\mathrm{ph} / 0506692$

[24] S. Nojiri and S.D.Odintsov, Phys.Lett.B 631 (2005)1.

[25] S.K.Srivastava, astro-ph/0511167.

[26] K.S.Stelle; Phys.Rev.D, 30 (1977) 953; E.S.Frandkin \& A.A.Tseylin; Nucl. Phys.B, 201 (1982) 469; E.Tombonlis; Phys.Lett.B, 70 (1977) 361; B.S.DeWitt; Phys.Rev., 160 (1967) 113; Ya.B.Zeldovich \& I.D.Novikov; Relyativisteviakaya Astrofizika (Relativistic Astrophysics), Fizmatgiz (1968); Ya.B.Zeldovich \& L.P.Pitaeveski; Comm.Math. Phys., 27 (1971) 185; V.Ts.Gurovich \& A.A.Starobinsky; Zh.Eksp.Teor.Fiz., $\quad 73$ (1977) 369 [Sov.Physa.JETP,46 (1977) 193]; B.Whitt;Phys.Lett.B, 145 (1984) 176; S.W.Hawking \& J.C.Luttrell; Nucl.Phys.B, 247 (1984) 250. 
[27] A.A.Starobinsky;Phys.Lett.B, 91 (1980) 99; Phys.Lett.B, 157(1985) 361; A.A.Kofmann, A.D.Linde \& A.A.Starobinsky;Phys.Lett.B, 157 (1985) 361.

[28] S.K.Srivastava and K.P.Sinha; Phys.Lett.B, 307 (1993) 40; Pramana, 44 (1993) 333; Jour. Ind. Math. Soc.61,80 (1994); Int.J.Theo.Phys., 35 (1996) 135; Mod.Phys.Lett.A, 12 (1997) 2933; S.K.Srivastava; Il Nuovo Cimento B, 113 (1998) 1239; Int.J.Mod.Phys.A, 14 (1999) 875; Mod.Phys.Lett.A, 14 (1999) 1021; Int.J.Mod.Phys.A, 15 (2000) 2917; Pramana, 60 (2003) 29.

[29] S.K.Srivastava, hep-th/0404170; gr-qc/0510086.

[30] A.D. Miller et al , Astrophys. J. Lett. 524 (1999) L1; P. de Bernadis et al , Nature (London)400 (2000) 955; A.E. Lange et al , Phys. Rev.D63 (2001) 042001; A. Melchiorri et al , Astrophys. J. Lett. 536 (2000) L63; S. Hanay et al , Astrophys. J. Lett. 545 (2000) L5.

[31] A.B. Lahnas, N.E. Mavromatos and D.V. Nanopoulos, Int. J. Mod. Phys. D, 12(9), 1529 (2003).

[32] A. Melchiorri, L.Mersini, C.J.Odman and M.Trodden, Phys.Rev.D, 68 (2003) 043509.

[33] N.D.Birrel and P.C.W.Davies, Quantum Fields in Curved Space (Cambridge Univ. Press, Cambridge,1982).

[34] A.R.Liddle and S.M.Leach, Phys. Rev. D 68, 103503 (2003); S.M.Leach and A.R.Liddle, Phys. Rev. D 68, 123508 (2003) ; Mon. Not. R.Astron. Soc. 341, 1151 (2003).

[35] E. Costa et al., Nature, 387,783 (1997); J.van Paradijs et al., Nature, 386, 686 (1997); A.D.Frail et al., Nature, 389, 261 (1997)

[36] J.S.Bloom et al., Astron. J., 123, 1111 (2002).

[37] G. Cusumano et al., astr0-ph/0509737.

[38] J.J.Halliwell, Phys.Lett.B 185, 341(1987); J. Yokoyama and K.-I. Maeda, Phys.Lett.B 207, 341(1987); A.A.Coley et al., J.Math.Phys. 385256 (1997); V.D. Ivashchuk, V.N.Melnikov and A.B.Selivanov, JHEP 0309, 059 (2003); T. Barreiro, E.J.Copeland and N.J.Nunes, Phys.Rev. D 61127301 (2000) ; A.A.Sen and S.Sethi,Phys.Lett.B 532, 159(2002), U.Franca and R.Rosenfield, JHEP 0210, 015 (2002).

[39] I.P.Neupane, hep-th/0311071. 